\title{
Spectral Density Method of Liquid Propellant Gauging
}

\author{
Celestine Ananda \\ NASA Kennedy Space Center
}

\begin{abstract}
A main objective of the Tank Health Monitoring group in the NASA Kennedy Space Center's Advanced Engineering Development Branch is to collect and process data to aid in developing Modal Propellant Gauging (MPG) for use in future Orion/SLS missions. The MPG project is an effort to develop a non-invasive low-cost propellant mass gauging technology for application to existing spacecraft propellant tanks in both low-gravity and earth-loading applications. A fuel gauging framework is developed and presented through characterizing the modal signature of a compositeoverwrap liquid propellant tank used for testing purposes. This is accomplished through presenting algorithms that apply broadband white noise signals to piezoelectric actuators adhered to the tank surface, processing acoustic data using Fourier analysis, and presenting frequency response functions to experimentally determine resonant modes of the tank structure. A limitation of the current MPG technique is that it does not work in environments with changing pressure. The Spectral Density Propellant Gauging Method is a technique that does not depend on the stiffness of the tank walls and is presented in this paper.
\end{abstract}

\section{Introduction}

Propellant is the primary limiter of spacecraft lifetime. Direct volume measurement using traditional buoyancy and level techniques are rendered ineffective in the microgravity environment of space. Indirect methods are used instead, however these methods typically incur considerable additional mass and expense to missions. Typical methods of propellant gauging also suffer from decreasing accuracy as the tank empties, rendering mass gauging accuracy lowest at the end of mission life when gauging accuracy is the most important.

Low-gravity gauging technologies include those that depend on bookkeeping, equations of state, and thermal mass estimation. Bookkeeping methods employs low rate monitors or, often, just assumptions about low rates during thruster rings, to estimate the total amount of propellant consumed over time. Such methods accumulate error over time and therefore introduce growing uncertainties in the estimate of remaining propellant mass. Equation of State methods rely on the inference of the liquid volume fraction within a tank pressurized by an external pressurizing gas (usually, Helium) based on measurements of pressure and temperature and the application of the ideal gas law (or other appropriate equation of state) to the ullage gas. In the PVT approach, the tank is considered isothermal, and method accuracy depends in part on the validity of this assumption. As in the case of bookkeeping methods, propellant mass estimates derived from applications of PVT methods are increasingly inaccurate at lower fill fractions. Thermal mass estimates rely on measurements of the temperature change induced in a propellant tank by external heating. Thermal response is compared to simulation results based on a tank-specific thermal capacitance model computed over a range of fill fractions. The uncertainty of propellant mass estimates based on TPG methods depend sensitively on the sophistication of the tank thermal model. In addition to low-gravity methods, settled propellant levels may be estimated using capacitance probes and various versions of impedance sensors. These 
electrical measurements are performed on settled liquids and are therefore used only during thrusting maneuvers and are subject to error induced by adhered liquid on sensors. Each family of methods has relatively reduced accuracy at low tank fill fractions and therefore propellant gauging. Error estimates on most gauging methods range from $1 \%$ to $>20 \%$. Adding additional propellant mass to account for gauging errors can result in significant launch costs and/or reduced mission life. The Modal Propellant Gauging (MPG) approach has the advantage of increased accuracy at low propellant fill fractions, is effective in both settled and sloshing propellants, and does not employ invasive hardware. Additionally, lab and parabolic flight testing suggest that the MPG method has gauging resolutions that are better than bookkeeping and PVT methods and comparable to the best TPG methods at low fill fractions, with gauging resolutions approaching $1.0 \%$ for settled propellant and $1.5-2.0 \%$ for unsettled (sloshing) propellants.

The MPG project is a collaboration between Carthage Space Sciences and Kennedy Space Center Advanced Engineering Development Branch to develop a high-resolution, low-gravity, fuel gauging technology that exploits the effect of fluid loading on the structural properties of liquidfilled propellant tanks. The technology that has been developed is flight tested through parabolic flight campaigns and suborbital flights onboard Blue Origin's New Shepard. This technology is being developed for implementation in the Orion/SLS Artemis Mission architecture.

\section{Concept}

The concept behind the Modal Propellant Gauging technology is based on analyzing resonance frequencies of fluid-filled tanks. The surface of a liquid in microgravity does not settle as it would on Earth; the configurations of liquid surfaces in microgravity environments are driven by tension and capillary forces. Because of this, in microgravity environments liquid adheres to the inner walls of the fuel tanks. The Modal Propellant Gauging technique takes advantage of the variable thickness of the liquid level on the walls of a tank.

Experimental Modal Analysis (EMA) involves recording the vibration spectrum of a solid object and using the spectral characteristics to infer the structural properties of the object. The EMA technique requires that all acoustic resonances in the tank structure are stimulated by applying a broadband white noise signal. Tank response is measured at discrete locations on the surface of the tank and the Fourier Transforms of the response and input functions are computed in realtime. Finally, the Frequency Response Function (FRF) for each structural response is computed by taking the ratio of the response FFT to the input FFT. The resulting FRF is a complex-valued function in which the real part is a function of the effective mass of the vibrating object and the imaginary part is related to the rate of energy dissipation through structural damping. Adding fluid to a tank lowers the real part of the frequency by increasing the effective mass. In addition, fluid in a closed tank changes the imaginary part of the frequency by damping the vibrations. 
3. Project Background Carthage College undergraduate students have been developing the Modal Propellant Gauging technology since 2008. There have been multiple versions of the payload, each with additional features to further advance the accuracy of the technology. Students have flown with Dr. Kevin Crosby on parabolic flights with NASA and the Zero-G corporation.

For the past two years author Celestine Ananda has functioned as the MPG Project Lead at Carthage College. Through this experience she became familiar with the MPG analysis approach and refined algorithms that determine liquid fuel volume in the propellant tanks. Her involvement with and contributions towards this project lead to her selection as an intern with the NASA Kennedy Space Center to develop and finalize the MPG algorithms for future use on the Orion crew capsule.

\section{Modal Propellant Gauging at the NASA Kennedy Space Center}

\subsection{Experiment Setup At the NASA Kennedy} Space Center, Celestine and intern Ethan Woller used a data acquisition unit (DAQ), a white noise generator, an amplifier, a scale, and a small linerless composite tank fitted with 5 piezoelectric (PZT) sensors to perform modal analysis, as shown in Figure 1. Sensor 1 functioned as the actuator, exciting the walls of the tank. Sensor 3 functioned as a monitor, recording the input signal and Sensor 4 served as a true sensor, recording the input signal and the modal response of the tank. The tank was filled in discrete 5\% increments and data was written from the PZT sensors to MATLAB to later perform FRFs. Once the tank had been filled the fluid was drained into a large bucket atop a scale at a continuous rate and the time-stamped scale readings were written to MATLAB. The time-stamped raw sensor data was aligned with the scale data and used in the analysis discussed in Section 4.2.

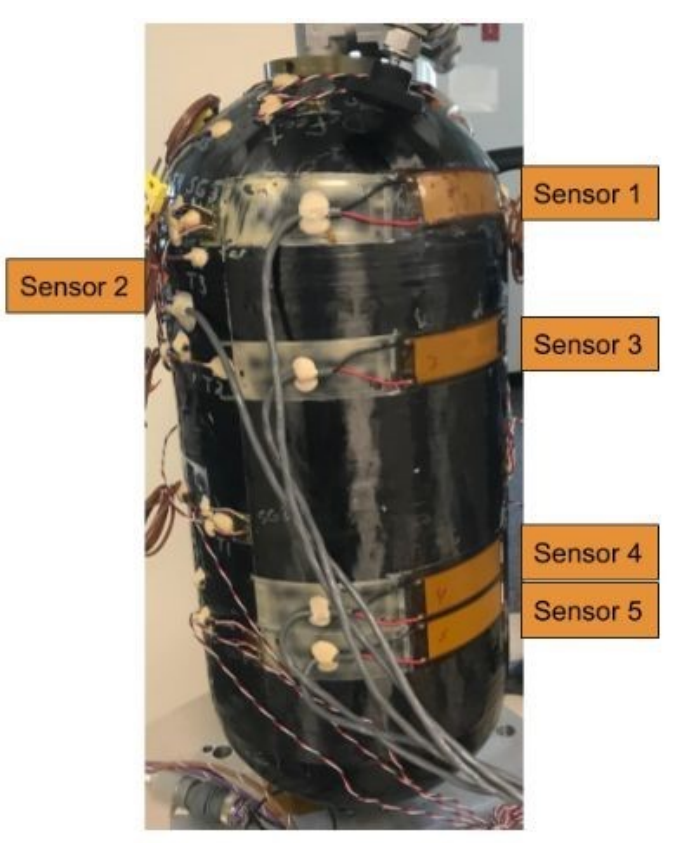

Figure 1. Tank Diagram

4.2 Estimating Propellant Mass from Discrete Fill Calibration Curve-Fit To perform modal analysis on the tank, MATLAB programs were developed to collect data from the DAQ, perform FRFs over varying durations of time, and to average the FRFs to reduce noise. It was found that the lowest mode of the empty tank is around $1050 \mathrm{~Hz}$ and the lowest mode of the tank when filled is around $500 \mathrm{~Hz}$, shown in Figure 2. A MATLAB program was developed to find the lowest mode frequency responses for 20 different fill fractions at 5\% tank volume increments. The peak frequencies and corresponding fluid masses were used to generate a log-log mass-frequency plot to produce a calibration curve to estimate tank fluid mass during a continuous drain. To produce the calibration curve, the equation of the linear best-fit was generated and produced from the log-log mass-frequency plot of a discrete fill in 5\% increments for FRFs. In this linear fit the dependent 
variable represents the logarithm of frequency and the independent variable represents the logarithm of mass.

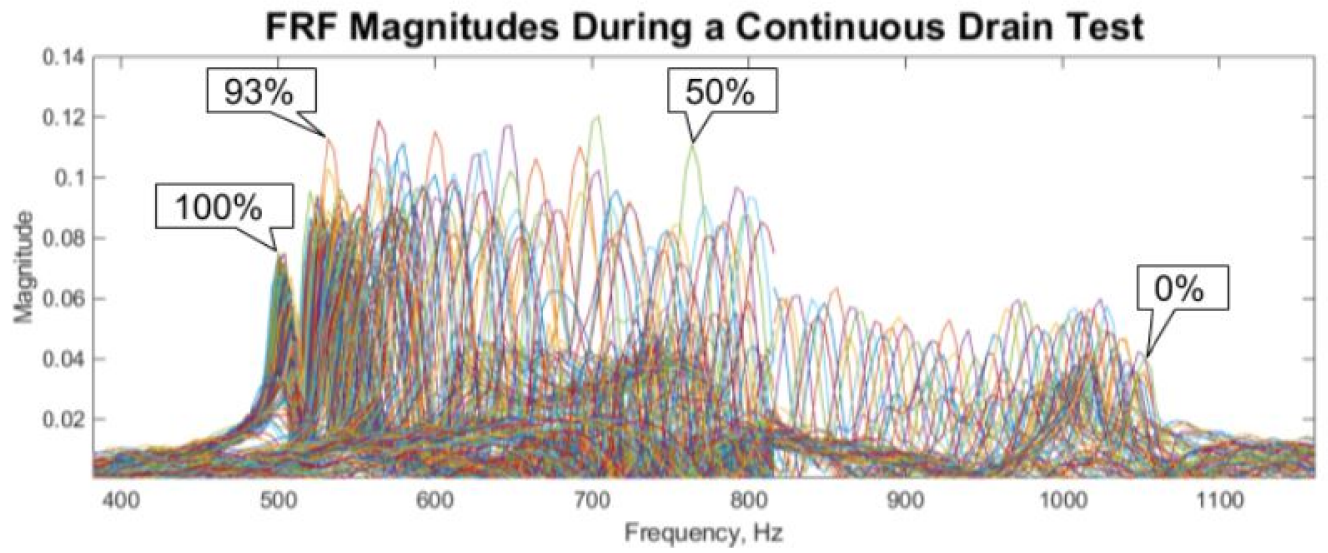

Figure 2. Processed FRFs, each averaged over 3 seconds in one second intervals during a contimuous drain test

Data from a continuous drain test was collected and processed to produce 1-second FRFs and find corresponding peak frequencies and actual fluid masses to test the resolution of the calibration curve. The log of peak frequency for each fill level was processed and substituted as the dependent variable in the calibration curve equation. This equation was then solved for the dependent variable, the log of the estimated mass. The logarithm base, 10, was then raised to this value to determine the estimated mass. In Figure 3, the estimate masses can be seen as the orange dots and the blue line represents the true mass of the system. The domed portion of the tank covers the first ten pounds (indicated via yellow line) and the last ten pounds (indicated via purple line). These portions are mentioned because the mass estimates in these regions are not accurate due to the change in tank geometry. The average resolution of this fit is $+/-3.18 \%$, or $+/-1.59 \mathrm{lbs}$. When only considering the region between $10-40$ $\mathrm{lbs}$, the average resolution is $+/-2.32 \%$, or $+/-1.16 \mathrm{lbs}$. 


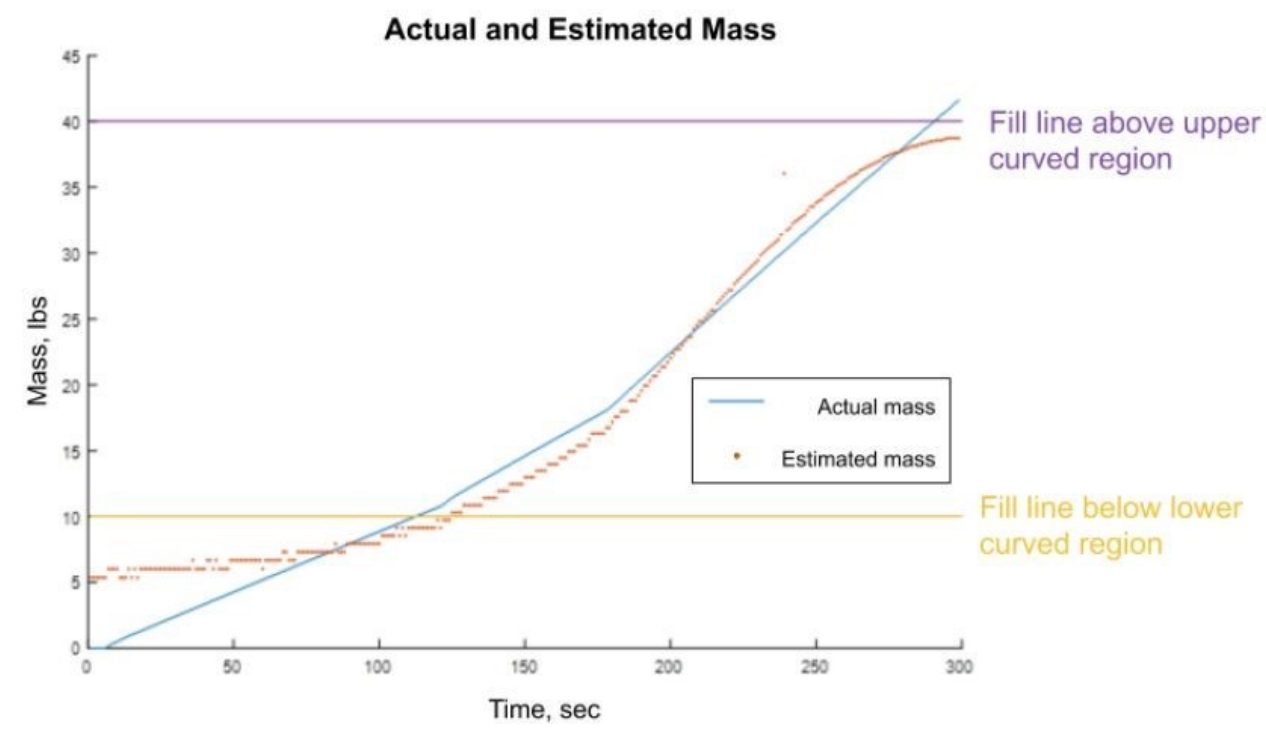

Figure 3. Actual and estimated mass of fluid within tank during a continuous drain test

\section{Effects of Changing Pressure}

Frequency is proportional to stiffness and mass. The MPG approach relates shifts in resonant frequencies to shifts in contained mass, which is accomplished through calculating modal masses and their participation factors. However, if the stiffness of the tank is changing, the frequency will shift regardless of effective mass. In application, the stiffness of the tank changes when the internal ullage pressure of the tank changes, typically during fill and drain operations. To determine the severity of this issue in regard to MPG peak tracking, tests were run with varying pressure in the linerless composite tank. The empty tank was pressurized to $\sim 200$ PSI (far below the maximum pressure rating) and modal data was collected at 197 PSI, 172 PSI, 140 PSI, 115 PSI, 50 PSI, and 0 PSI. From 197 PSI to 0 PSI, peaks shifted an average of $20 \mathrm{~Hz}$, as shown in Figure 4. This shift is large enough to render the peak-tracking MPG technique inadequate in fuel gauging applied to linerless composite tanks changing in pressure. This complication is remedied through the MPG Spectral Density Method. 


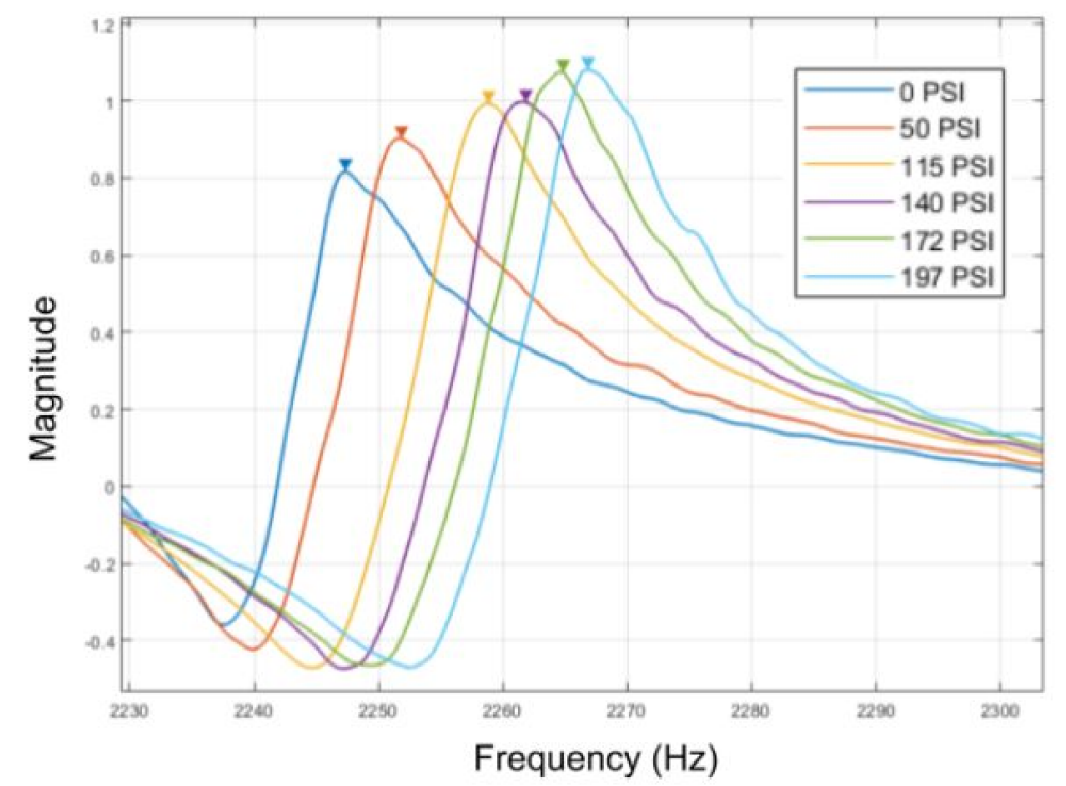

Figure 4. A graph depicting a mode of the tank changing in frequency as pressure changes and fluid volume remains constant.

\section{Spectral Density Method}

The Spectral Density Method (SDM) of liquid propellant gauging is an EMA technique that does not depend on the stiffness of the tank, only depending on modal mass.

6.1 Spectral Density Function The "spectral density" is the Fourier Transform of a correlation function of a stationary, random input signal [1]. For a system defined by a single input signal $x(t)$ and a single output signal $y(t)$, the spectral densities of the input and output signals $S_{x x}(w)$ and $S_{y y}(w)$ are related via the FRF, $H(w)$,

$$
S_{y y}(w)=\int_{0}^{\infty}|H(w)|^{2} S_{x x}(w) d w
$$

In MPG, the input signal, $x(t)$, is a broadband white noise signal, for which the input spectral density is a constant, thus $S_{x x}(w) \equiv S_{0}$. This constant is the average value of the input spectral density, therefore the output spectral density is

$$
S_{y y}(w)=S_{0} \int_{0}^{\infty}|H(w)|^{2} d w
$$

In other papers [1] it is shown that the second "moment" of the spectral density for broad-band excitations is related to the modal mass components of a single degree of freedom system that behaves like a set of $N$ coupled, damped linear oscillators through the following:

$$
S_{y y}^{2}(w)=S_{0} \int_{0}^{\infty} w^{2}|H(w)|^{2} d w=\frac{\pi}{2} \sum_{n=1}^{N} \frac{a_{n}^{2}}{M_{n}}
$$


where $\alpha$ is constant and $M_{n}$ is the modal mass of the $n^{\text {th }}$ mode. A visual representation of this integral applied to modal data is shown in Figure 5, and the results of this method applied to data obtained during a continuous drain of the composite linerless tank is shown in Figure 6, where the tank is treated as a single damped linear oscillator. The validity in treating the tank as a single damped linear oscillator is discussed in Section 6.

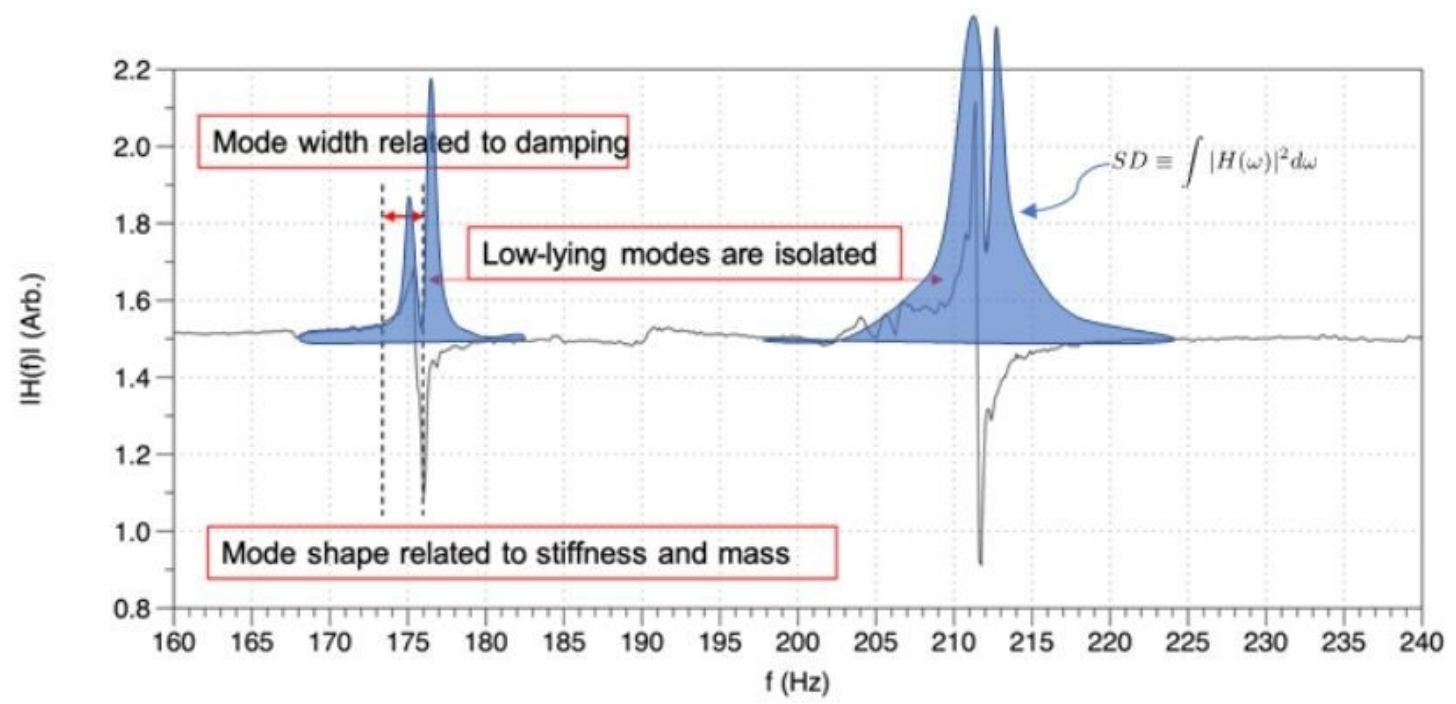

Figure 5. A visual depiction of the spectral density integral value applied to modal data. 


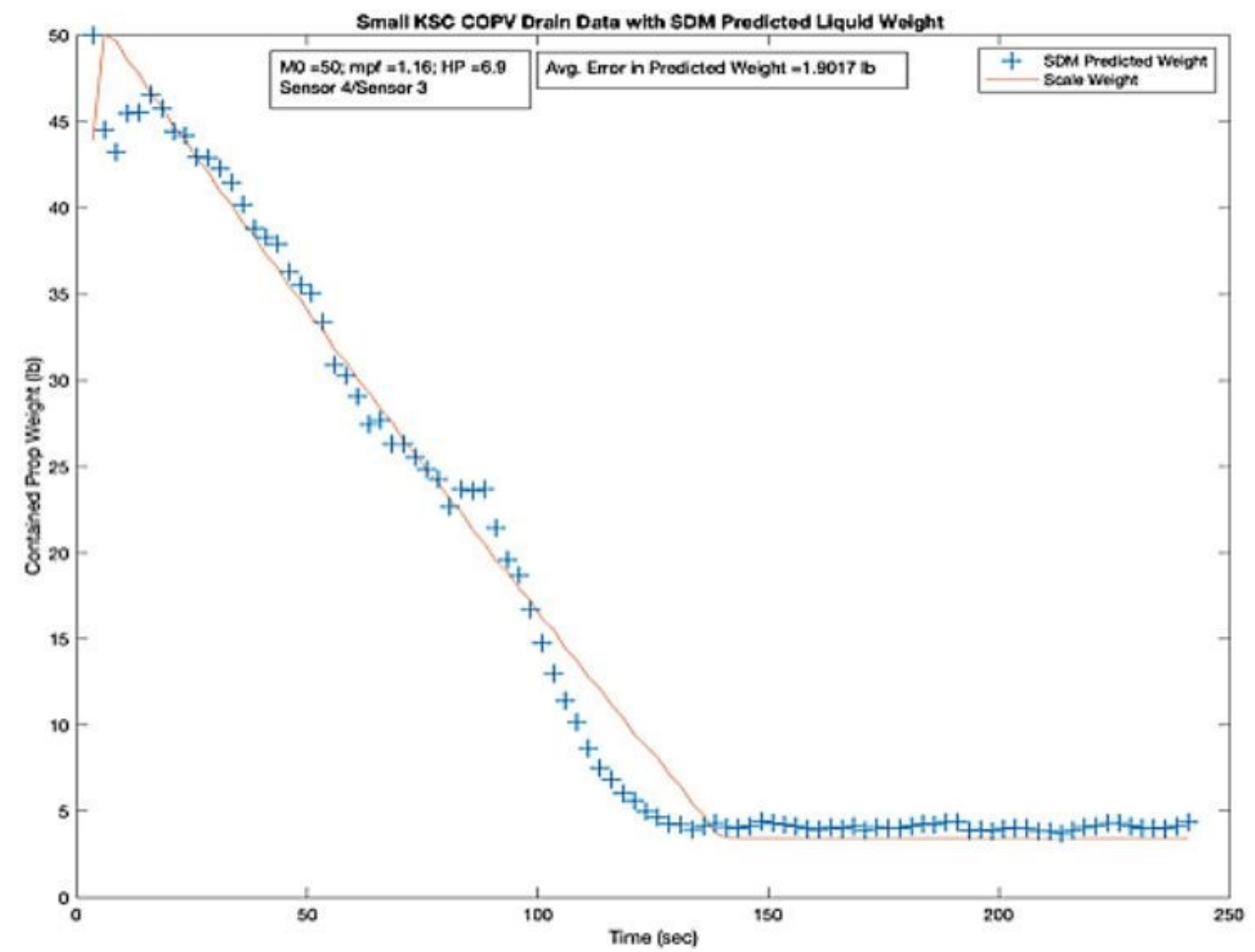

Figure 6. SDM applied to the composite linerless tank during a drain. The red line indicates the actual contained fluid mass and the blue marks indicate the SDM contained mass estimate.

\subsection{State Space Estimations The}

Spectral Density Method applied to propellant gauging follows the modal mass, not the actual contained mass. The difference between these values depends on how many modes are active in the tank's spectrum. Treating the propellant tank as a single, damped linear harmonic oscillator, in which only the lowest mode is tracked, simplifies analysis algorithms in that the modal mass and the contained mass are the same value. To ensure this is a fair approximation, a state-space representation of the tank was built in MATLAB. The combined results of this analysis and of finite element analysis proved that this is a fair approximation for this specific composite linerless tank. Results of modeling the tank as a single damped linear oscillator and applying SDM to this model as it "drained" is shown

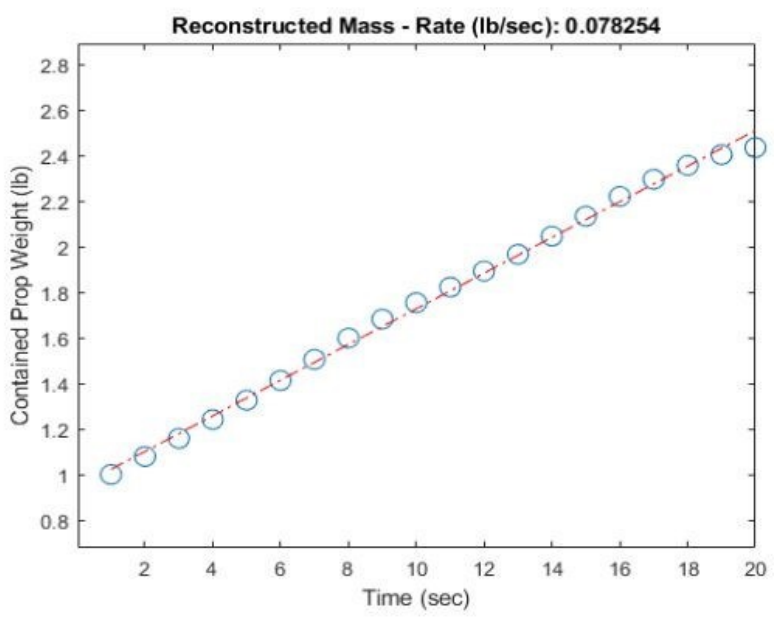

Figure 7. The result of applying SDM to a state space of this simplified system. 
in Figure 7, and the physical depiction of the tank as a harmonic oscillator is shown in Figure 8.

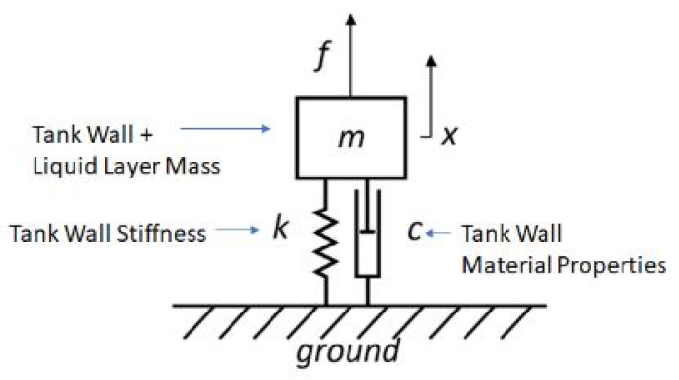

Figure 8. A visual depiction of what it physically means to model the tank as a single damped linear oscillator

\section{Orion Use Case Analysis}

The SDM-MPG technology is in consideration for propellant monitoring for future Orion/SLS missions. To assist in these considerations an Orion-Gateway mission profile was analyzed to determine the use-case of SDM-MPG within the mission structure.

7.1 Assumptions The following simplified analysis assumes the mission profile presented in "Options for Staging Orbits in Cis-Lunar Space", Ryan Whitley and Roland Martinez [2], represents current Orion plans. This analysis also assumes full Orion ESM prop tanks at first lunar flyby per Whitley/Martinez and assumes propellant and oxidizer drain at the same rate.

7.2 Analysis The mission profile outlines four burns and corresponding delta-v's: Flyby I $(178 \mathrm{~m} / \mathrm{s})$, Insertion $(250.5 \mathrm{~m} / \mathrm{s})$, Departure $(221.5 \mathrm{~m} / \mathrm{s})$, and Flyby II $(190 \mathrm{~m} / \mathrm{s})$. This lends a total Orion-Gateway EarthNRO transfer mission cost of $840 \mathrm{~m} / \mathrm{s}$. The burnprofile analysis assumes a total vehicle dry mass of $17000 \mathrm{~kg}$, a useable propellant mass of $8685 \mathrm{~kg}$, and a residual + contingency propellant mass of $5 \%$ of the total propellant mass $-434 \mathrm{~kg}$, for a total wet mass of $26119 \mathrm{~kg}$. This burn-profile will also assume an ISP of $315 \mathrm{~s}$, a maximum delta-V capability of $1250 \mathrm{~m} / \mathrm{sec}$, and a single engine thrust of $26089 \mathrm{~N}$. Resulting burn times and total mass of propellant burned during the four events was calculated through the rocket equation and is presented in

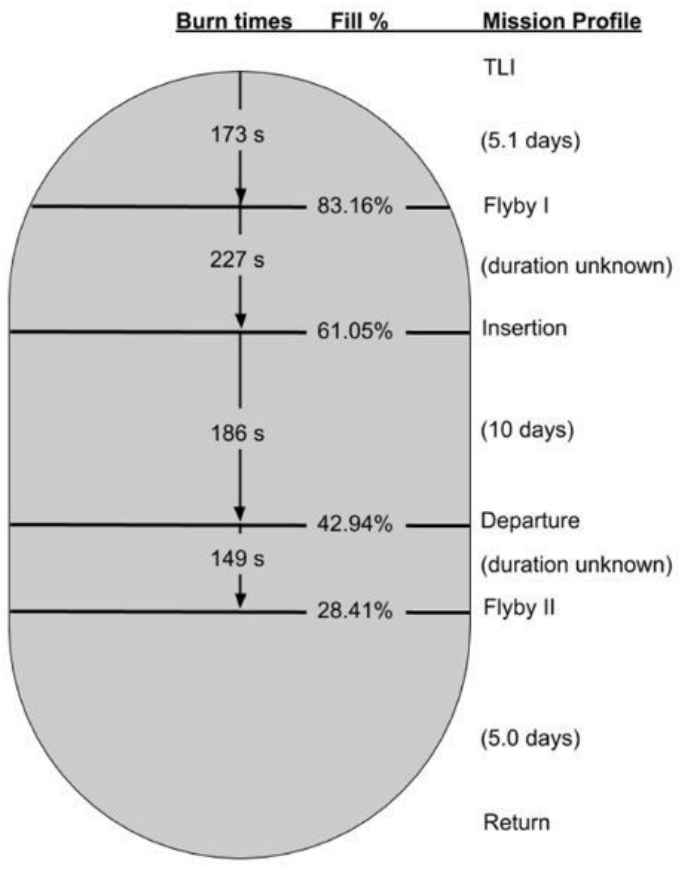

Figure 9. Mission Profile in relation to tank fill \% - Dimensions to scale with Orion tanks 
Table 1. These results are used to determine the burn rate per tank, $2.1 \mathrm{~kg} / \mathrm{sec}$. A representation of the mission profile, events, and corresponding burn durations in relation to tank fill fractions is shown in Figure

9.

\begin{tabular}{|c|c|c|c|c|}
\hline & Delta - V (m/sec) & Total Prop Burned (kg) & $\begin{array}{l}\text { Prop Burned/tank } \\
\text { (kg) }\end{array}$ & Burn Time \\
\hline Flyby I & 178.0 & 1462 & 365.5 & 173.2 \\
\hline Insertion & 250.5 & 1920 & 480.0 & 227.4 \\
\hline Departure & 221.5 & 1573 & 393.0 & 186.3 \\
\hline Flyby II & 190.0 & 1262 & 315.5 & 149.5 \\
\hline Total & $840.0 \mathrm{~m} / \mathrm{sec}$ & $6217 \mathrm{~kg}$ & 1554 kg & $736.4 \mathrm{sec}$ \\
\hline
\end{tabular}

Table 1. Propellant and Burn Time Profile

7.3 MPG-Orion Use Case Recommendation To generate a notional gauging plan for the mission profile, it is assumed that at least $1 \mathrm{~GB}$ of storage will be dedicated to modal/RMS data for each tank and 8 PZT sensors will be attached to each tank. A single cell of a CSV file contains 7 bytes of data, therefore 8 sensors collecting data at a sampling rate of 16384 samples/sec will write 917.56 KB per second. Each tank can then collect 1089 seconds of data if $1 \mathrm{~GB}$ of storage is dedicated to modal data for each tank. Data should be taken during burns to perform Root Mean Square Method calculations and data should be taken during static states between burns to perform SDM calculations. Collecting data during burns will require 737 seconds, leaving 352 seconds to take data during static states between burns. Static state SDM measurements should be made during settled states. Data should then be taken during the 5.1 day period between trans-lunar injection (TLI) and the first lunar Flyby to profile the behavior and frequency response of a full tank in zerog. Data could also be taken during static states at $83.16 \%, 61.05 \%, 42.94 \%$, and at $28.41 \%$. The 
remaining 352 seconds would allow for 70 seconds of data to be taken at each of the five static fill levels.

\section{Acknowledgments}

I would like to thank my research advisor and the director of WSGC, Kevin Crosby, for his support throughout my experiences at Carthage and at the NASA Kennedy Space Center. I would additionally like to thank the Wisconsin Space Grant Consortium for funding this project. Finally, I would like to thank Mr. Rudy Werlink for his mentorship and for providing this opportunity.

\section{References}

[1] Crosby, Kevin M., et al. "Modal Propellant Gauging: High-Resolution and Non-Invasive Gauging of Both Settled and Unsettled Liquids in Reduced Gravity." Acta Astronautica vol. 159, 2019, pp. 499-507., doi:10.1016/j.actaastro.2019.01.050.

[2] Whitley, R., \& Martinez, R. (2015, October 21). Options for Staging Orbits in Cis-Lunar Space. Retrieved July 1, 2019, from https://ntrs.nasa.gov/archive/nasa/. 